\title{
Universal features of water dynamics in solutions of hydrophilic polymers, biopolymers, and small glass-forming materials
}

\author{
Silvina Cerveny, ${ }^{1,2}$ Ángel Alegría, ${ }^{2,3}$ and Juan Colmenero ${ }^{1,2,3}$ \\ ${ }^{1}$ Donostia International Physics Center, Paseo Manuel de Lardizabal 4, 20018, San Sebastián, Spain \\ ${ }^{2}$ Centro de Física de Materiales, Centro Mixto CSIC-UPV/EHU, San Sebastian, Spain \\ ${ }^{3}$ Departamento de Física de Materiales, Facultad de Química, Universidad del País Vasco (UPV/EHU), Apartado 1072, \\ 20018, San Sebastián, Spain
}

(Received 23 March 2007; revised manuscript received 17 October 2007; published 17 March 2008)

\begin{abstract}
A systematic investigation by dielectric spectroscopy of 18 different water-rich mixtures with very different hydrophilic substances shows universal features for the water dynamics. The temperature dependence of the relaxation times exhibits a crossover from non-Arrhenius to Arrhenius behavior at the $T_{g}$ range of the mixtures. Furthermore, the temperature dependence of the relaxation times presents a universal behavior both above and below the crossover temperature. We also show that these features suggest that the observed crossover is associated with the emergence of confinement effects.
\end{abstract}

DOI: 10.1103/PhysRevE.77.031803

PACS number(s): 82.35.Pq, 61.25.Em, 77.22.Gm

\section{INTRODUCTION}

The behavior of water closely associated with-or restricted by-other molecules and systems is a subject of very active research $[1,2]$. The main driving force for these studies is that water in cells and living organisms is always linked to proteins and other biomolecules, and hydration seems to play a decisive role in controlling the structure, stability, and function of these systems $[3,4]$. Therefore understanding hydrated systems in general and how the dynamics of water affects or control the properties of these systems are emerging questions of utmost importance.

Water dynamics in solutions of polymers, small organic molecules, and biomolecules in the low-temperature range $(130-250 \mathrm{~K})$ have been investigated by different experimental techniques such as neutron scattering, dielectric spectroscopy, NMR, etc. [5-7]. In particular, by dielectric spectroscopy (DS) often two relaxation processes [5,8-10] are observed at water concentrations about 50 wt \% (we will call these processes I and II). The relaxation times of the slower process (I) usually follows a Vogel-Fulcher-Tammann [11] (VFT) temperature dependence, and it was considered to be due to the cooperative rearrangement of the whole system, i.e., the matrix (solute) plus water. The characteristics of this process are quite similar to those exhibited by the well known $\alpha$ relaxation, which is observed in supercooled systems above the glass transition temperature range $\left(T_{g}\right)$. On the other hand, the faster process (II) has usually been associated with the reorientation of water molecules in the solution $[5,8,12,13]$. The temperature dependence of these relaxation times is usually Arrhenius-like below the glass transition of the hydrated systems, suggesting that in this temperature range water motions are in some way restricted by the glassy matrix [14]. It is worthy of remark that at room temperature the complex dielectric permittivity $\varepsilon^{\prime \prime}$ of aqueous solutions also exhibit a bimodal relaxation process $[1,15,16]$, suggesting a connection between the two regimes.

However, in spite of these results, a systematic and comparative study of water dynamics in hydrophilic systems of very different nature still needs to be done. With these ideas in mind, in this work, we have investigated the water dynamics in different solutions of low-molecular-weight organic glass formers, polymers, sugars, and biopolymers (proteins and DNA). We found that, despite the fact that water in these systems is in principle surrounded by different environments and different molecular interactions between water and solute should take place, the dynamics of water in these systems show some universal features. In particular, the temperature dependence of the main relaxation time characterizing water dynamics (process II) is almost the same (within the experimental uncertainty) for all systems investigated both below and above the glass transition temperature of the system.

\section{EXPERIMENT}

We have selected different systems (which differ substantially in molecular weight), such as water-soluble polymers [poly(vinyl methyl ether) (PVME), poly(vinyl pyrrolidone) (PVP)], low-molecular-weight organic glass formers [pentaethylene glycol (5EG) and sorbitol], sugars (fructose, lyxose, and ribose), and biopolymers (myoglobin from horse heart and deoxyribonucleic acid (DNA) from herring testes]. We have considered the water concentration $\left(w_{c}\right)$ in the range between 30 and 55 wt \%, since in this concentration range the solute is completely hydrated and, as we will show below, self-association of water molecules is relevant for process II.

To study the water dynamics in these systems, we have carried out broadband dielectric spectroscopy in the frequency range $10^{-2}-10^{7} \mathrm{~Hz}$ and over the temperature range 140-250 K by means of a Novocontrol Alpha analyzer system. The experimental procedure is described in Ref. [13]. The samples were placed between parallel gold-plated electrodes with a diameter of $30 \mathrm{~mm}$. The sample thickness was typically $0.1 \mathrm{~mm}$. After cooling at a rate of $10 \mathrm{~K} / \mathrm{min}$, isothermal frequency scans recording the complex dielectric susceptibility $\varepsilon^{*}(\omega)$ were performed every $5 \mathrm{~K}$ over the temperature range $140-290 \mathrm{~K}$. The sample temperature was controlled with stability better than $\pm 0.1 \mathrm{~K}$. Since the water 
molecule has a big dipolar moment, dielectric spectroscopy is a very suitable technique to analyze the reorientational dynamical behavior of aqueous solutions in a broad frequency range. In addition to the systems described above and in order to generalize our results, data corresponding to other hydrated systems were taken from the recent literature (glucose [17]), poly(ethylene glycol) (PEG600, $M_{n}=600 \mathrm{~g} / \mathrm{mol}$; PEG200, $M_{n}=200 \mathrm{~g} / \mathrm{mol}$ ) [17], (propylene glycol and its oligomers, $n=1,2,3)$ ( $n \mathrm{PG}$ ) [8]. Moreover, high-temperature data for water solutions with myoglobin [1], DNA [18], PVP, and PVME [19] are also included for comparison. It is noteworthy that the $T_{g}$ values of these solutions span a rather broad range from 165 to $220 \mathrm{~K}$.

Finally, a differential scanning calorimeter (DSC) TA Instrument Q1000 was used in standard mode to control the crystallization of the samples. Standard DSC measurements were performed using cooling and heating rates of $10 \mathrm{~K} / \mathrm{min}$. Hermetic aluminum pans were used for all the materials. The sample weights were about $10 \mathrm{mg}$. In the water mixtures of polymers, sugars, and low-molecular-weight organic glass formers, there is no evidence of crystallization on cooling. In the case of DNA and myoglobin only about $5 \%$ of the water in the solutions crystallizes on cooling at $\sim 235 \mathrm{~K}$. Furthermore, all the mixtures show on heating a pronounced calorimetric glass transition, indicating that there is a main glass phase at low temperatures. Therefore, we have been able to study the dynamics of water in these systems in both the supercooled liquid and the glassy state.

\section{RESULTS AND DISCUSSION}

The dielectric spectra of all the systems analyzed present the two main relaxation processes above mentioned: the slower process I (we will denote this process as PI) and the faster process II (it will be denoted as PII). A representative example for 5EG is shown in Fig. 1. Whereas PI was fitted by a Havriliak-Negami function [20], PII is symmetric and was described by the empirical Cole-Cole equation [21]. The broadening of PII results in the range of 2-3.5 decades for all the materials analyzed. Inset 1 in Fig. 1 shows how the relaxation strength of PII $\left(\Delta \varepsilon_{\text {II }}\right)$ calculated from this fitting increases more rapidly at high $w_{c}$. This indicates an increase in the water mobility, suggesting the appearance of water domains. Thus, in the high-water-concentration range, waterwater interactions would be dominant for the relaxation mechanism of PII [12]. This could indicate a microheterogeneous structure, where water-rich domains of significant size are present. This picture is supported by recent molecular dynamics (MD) simulations of closely related systems that postulate the existence of water cluster in solutions of PVP [22], glucose [23], and fructose [24]. Moreover, the frequency-dependent dielectric properties of ubiquitin solution were recently calculated from MD simulations. The dielectric response was decomposed into protein-protein, water-water, and water-protein cross terms [25] and the fastest process was found to arise from the self-term of water. This is in agreement with our finding at lower temperatures.

In order to obtain model-independent experimental results, the main relaxation times corresponding to PI and PII

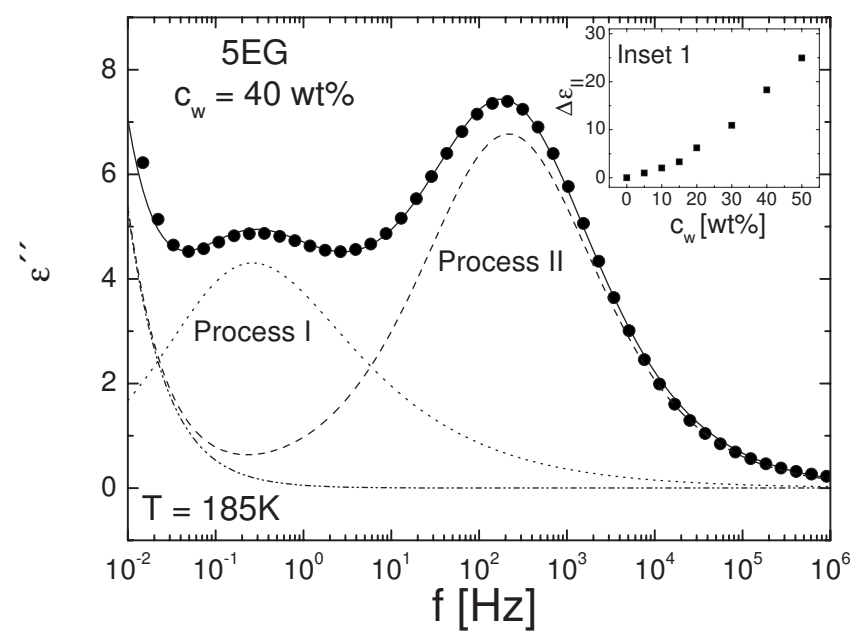

FIG. 1. Loss component $\boldsymbol{\varepsilon}^{\prime \prime}$ of the complex dielectric permittivity of a 5EG-water solution at $185 \mathrm{~K}$. The solid line through the data points is a least-squares fit using a superposition of a power law for conductivity, a Harriliak-Nagami function for process I, and a Cole-Cole (CC) function for process II. Inset 1: Variation of the relaxation strength with water concentration $\left(w_{c}\right)$ for 5EG-water mixtures. A dramatic increase is noted for high water concentration $\left(w_{c}>20\right.$ wt $\left.\%\right)$.

were simply obtained from the corresponding maxima of the dielectric loss spectra $\varepsilon^{\prime \prime}(\omega)$ at constant temperature as $\tau$ $=1 / \omega_{\max }$. As an illustrative example, Figs. 2(b) and 2(d) show the temperature dependence of the relaxation times obtained in this way in the case of a solution of 5EG [Fig. 2(b)] and a solution of PVME [Fig. 2(d)], both at a water concentration $\left(c_{w}\right)$ of $40 \mathrm{wt} \%$. The temperature dependence of the relaxation times corresponding to PII exhibits a crossover
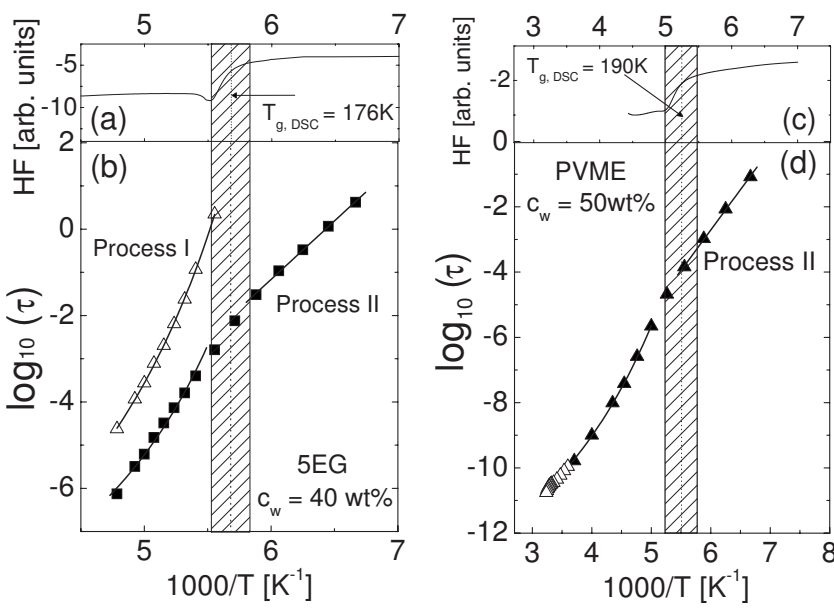

FIG. 2. (a), (c) Heat flow measured by DSC of 5EG-water solution and PVME-water solution during heating at a rate of $10 \mathrm{~K} / \mathrm{min}$. (b), (d) Temperature dependence of the relaxation times obtained from dielectric spectroscopy on 5EG-water and PVMEwater solutions. Data for PVME in the high-frequency and temperature range are shown as open points [19]. Note that for polymeric solutions only PII is resolved due to the fact that the dc conductivity as well as the electrode polarization contributions mask the slower and likely much weaker PI. 
from a non-Arrhenius (Vogel-Fulcher-like) behavior to an Arrhenius dependence as the temperature decreases [14]. Figures 2(a) and 2(c) clearly demonstrate that this crossover takes place in the temperature range where differential scanning calorimetry measurements on the same samples show the global glass transition process. This is a general trend for all systems investigated. Thus, the crossover observed should be associated with the freezing of the mixture at the glass transition temperature. It is worthy of remark that in the systems where PI can be experimentally resolved, the relaxation time of this process takes values of about $100 \mathrm{~s}$ in the $T_{g}$ range, i.e., in the range where the crossover of PII is observed. This again supports the above-mentioned interpretation of PI as representative of the global dynamics of the system.

In the framework of the above results, we can interpret the crossover displayed by process II in the following way. As has already been mentioned, PII has been associated with water-water interactions in the mixtures. At high temperature, above $T_{g}$, we can expect that water reorientation is coupled to the cooperative motions involved in the global $(\alpha$-like) relaxation process of the system. As soon as the temperature is decreased toward $T_{g}$, the global dynamics becomes frozen, but water molecules still have a significant mobility to be detected in the dynamic window of dielectric spectroscopy. Below $T_{g}$, water molecules are in some way trapped in a frozen matrix and thereby their motions have to be restricted and similar to those corresponding to a secondary $\beta$ process in a simple glass. As a consequence, the temperature dependence of the relaxation times is Arrheniuslike. This interpretation is supported by the fact that water dynamics in well-defined geometrical confinements such as molecular sieves [26] and purple membrane [27] seems to show a similar crossover. In addition, for PVP-water mixtures, the formation of clusters or strands of water molecules occupying channels between the polymer chains was revealed by MD simulations at low temperatures [22]. To our knowledge there are not similar literature data for the other studied materials. Of course, in the case of the mixtures we are discussing here, the "confinement" length would be ill defined. It is also worth mentioning that this kind of crossover from liquidlike dynamics towards confinedlike behavior has also been reported recently for the dynamics of the fast component in asymmetric polymer blends, i.e., blends where the two components exhibit different mobilities [28-31]. This suggests the possibility that this crossover is a general trend of any dynamical asymmetric mixture. Whether or not this crossover is related to the viscoelastic phase separation predicted for such systems (see, e.g., Ref. [32]) is a question that needs further investigation beyond this work. Another system where the appearance of such a crossover was observed is ionic conductors[33] where again the ion mobility is much higher than the matrix mobility, supporting the generality of this scenario.

When we compared the Arrhenius temperature dependence of water dynamics (PII) in the glassy state for all systems here considered (including water in molecular sieves [26] and purple membrane [27]), we found very similar activation energies. In fact an almost constant value-within the experimental uncertainty $-E_{a}=(0.54 \pm 0.04) \mathrm{eV}$ can be
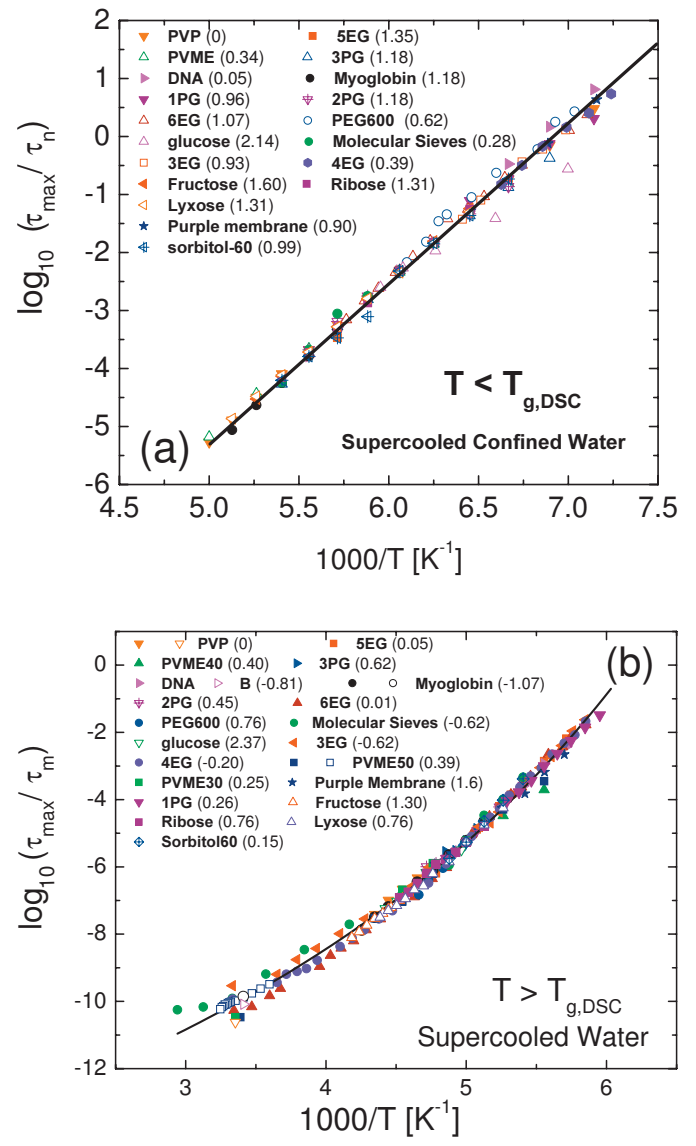

FIG. 3. (Color) (a) Master curve of the dielectric relaxation time for water dynamics in a wide variety of systems at temperature lower than $T_{g \text {,DSC }}$. The number in parentheses $\left(\tau_{n}\right)$ indicates the vertical shift applied to each system. Biological systems: myoglobin (this work, 44 wt \%), DNA (this work, $30 \mathrm{wt} \%$ ), and glucose (33 wt \%) [17]. Sugars: fructose (this work, $30 \mathrm{wt} \%$ ), lyxose (this work, $30 \mathrm{wt} \%$ ), ribose (this work, $30 \mathrm{wt} \%$ ), and glucose (35 wt \%) [17]. Polymeric systems: PVME (this work, 40 and 50 wt \%) and PVP this work, 30, 40, and 55 wt \%. Other synthetic systems: sorbitol (this work, $40 \mathrm{wt} \%$ ), 3EG triethylene glycol (3EG) [5], tetraethylene glycol (4EG) [5], pentaethylene glycol (5EG) [5], hexaethylene glycol (6EG) [5] (35 wt \%), nPG $(n$ $=1,2,3)$, [4] (40 and $50 \mathrm{wt} \%)$, PEG600 (33 wt \%). In addition we also show the results for water geometrically confined in molecular sieves [26] and purple membrane [27]. The solid line represents the Arrhenius equation with $E_{a}=0.54 \mathrm{eV}$. (b) Master curve of the dielectric relaxation time for hydration water dynamics in a wide variety of systems at temperature higher than $T_{g}$. The solid line represents the VFT equation (see text). The number in parentheses $\left(\tau_{m}\right)$ indicates the vertical translation applied to each system [symbols as in (a)] Literature data at room temperature for PVP, PVME [19], DNA [18] and myoglobin [1] are also included.

deduced. This implies that a master curve for the temperature dependence of water dynamics below the crossover temperature could be obtained by properly shifting the relaxation times of all systems on the $Y$ axis. The master curve so obtained is shown in Fig. 3(a) and summarizes the universal behavior of water dynamics (PII) in 20 systems of very different nature. Note that the universal value for $E_{a}$ is larger than that found for water dynamics in cases where almost 
individual molecules are expected to be involved $(0.47 \mathrm{eV}$ for the diffusion of water in glassy water and $0.46 \mathrm{eV}$ for solutions with very low $w_{c}[6]$ ). This observation is in agreement with the interpretation of self-association of water molecules in hydrophilic solutions at higher $w_{c}$.

Now we can ask what the situation is concerning the temperature dependence of PII above the glass transition of the system. In this range, the mixture is in a supercooled liquidlike state and thereby the temperature dependence of the relaxation times is non-Arrhenius as has already been mentioned. Astonishingly, the data in this range corresponding to all systems here considered can also be collapsed onto a master curve. This is shown in Fig. 3(b). This finding shows that the universality of the temperature dependence of water dynamics in (relatively rich) mixtures with hydrophilic substances holds both below and above the crossover range. These results support the idea that self-association of water molecules is mainly responsible for process II. This master plot can be used to estimate the parameters of a VFT equation, $\tau \propto \exp \left[B /\left(T-T_{0}\right)\right]$ where $B=D T_{0}$ and $D$ is related to the fragility index $m$ first introduced by Angell to classify the temperature behavior of supercooled liquids [34]. The calculated value of $T_{0}$ was $96 \pm 5 \mathrm{~K}$. This $T_{0}$ can be fixed and then used to calculate the VFT parameters for each investigated mixture. An average value of $B=2393 \pm 362 \mathrm{~K}$ can be deduced. From $B$ and $T_{0}$ a value of the fragility parameter $m$ $=40 \pm 5$ is obtained $\left(\mathrm{m}\right.$ is the slope of the $\log _{10} \tau$ vs $T_{g} / T$ curve at $T_{g}$ ). This value suggests that water in hydrophilic mixtures above $T_{g, \text { DSC }}$ seems to behave as a relatively strong glass former [35] in this temperature regime. Note that the universality is noticeable in the temperature dependence of the relaxation times, but not in the absolute value of the relaxation times. Both the absolute value of the relaxation times and consequently the value of $\tau_{0}$ in the VFT equation vary by about two orders of magnitude depending on the solution considered. This disparity in the values of $\tau_{0}$ question brings into the interpretation of $\tau_{0}$ as a molecular vibration time and more likely suggests the existence of a new crossover of the temperature dependence of the water relaxation time at higher temperatures and frequencies

From the above results it follows that the observed universalities confirm that process II is entirely driven by water molecules. On the other hand, it is known that the dynamics and functions of a protein solution can be slaved to motions in the bulk solvent and the hydration shell [36]. The question is whether this behavior is also true for other solutions. Fig-

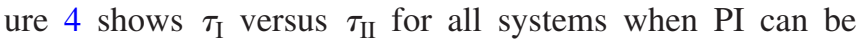

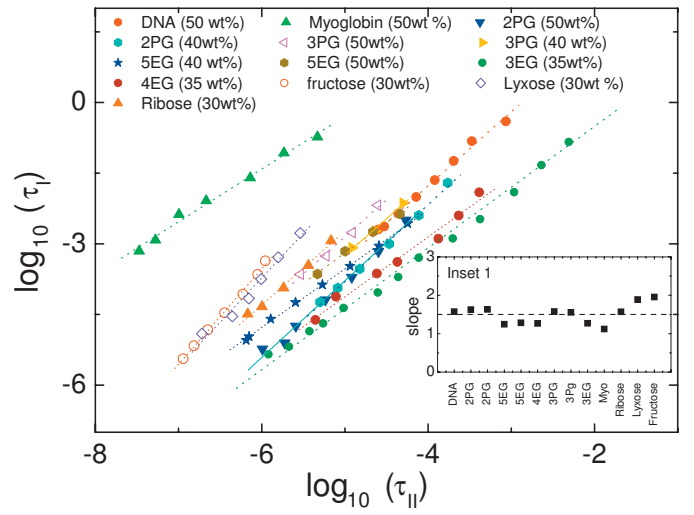

FIG. 4. (Color) Correlation between the relaxations times for process I and process II at temperatures higher than $T_{g}$. The number in parentheses indicates the corresponding water content.

experimentally resolved. A power law relationship between $\tau_{\mathrm{I}}$ and $\tau_{\mathrm{II}}$ is observed for all systems considered, which indicates a correlation between processes I and II. However, whereas the slope of the line is 1 for proteins [36-38] (direct proportionality of $\tau_{\mathrm{I}}$ and $\tau_{\mathrm{II}}$ ), it is higher for the rest of the systems analyzed (see inset 1 in Fig. 4). However, a slope different from unity does not indicate a close correlation between the water and solute dynamics, but it is an indication that water does not drive completely the dynamics of PI for all the solutions.

\section{CONCLUSION}

In conclusion, by considering water solutions of 18 systems of very different physical nature we have demonstrated that the temperature dependence of the relaxation time of water dynamics displays a crossover for non-Arrhenius to Arrhenius behavior of the global glass transition of the system. This crossover seems to be a general feature not only for water solutions but for dynamically asymmetric mixtures. Nevertheless, our main finding is that the temperature dependence of the relaxation times characterizing the dynamics of water in mixtures with hydrophilic substances is universal both below and above the crossover.

\section{ACKNOWLEDGMENTS}

The authors acknowledge the support of the University of Basque Country and the Basque Government, Project No. UPV/EHU, 9/UPV 00206.215-13568/2001 and the Spanish Ministry of Education Project No. MAT2007-63681.
[1] N. Nandi, K. Bhattacharyya, and B. Bagchi, Chem. Rev. (Washington, D.C.) 100, 2013 (2000).

[2] M. C. Bellissent-Funel, J. Phys.: Condens. Matter 13, 9165 (2001).

[3] A. V. Finkelstein and O. B. Ptitsyn, Protein Physics (Academic Press, London, 2002), Vol. 1, Chap. 6, p. 57.

[4] H. Flyvbjerg and O. G. Mouritsen, Physics of Biological Systems: From Molecules to Species (Spring-Verlag, Berlin,
1997), Vol. 1, p. 29.

[5] S. Sudo et al., J. Chem. Phys. 121, 7332 (2004).

[6] S. Cerveny, G. A. Schwartz, R. Bergman, and J. Swenson, Phys. Rev. Lett. 93, 245702 (2004).

[7] H. Jansson, W. S. Howells, and J. Swenson, J. Phys. Chem. B 110, 13786 (2006).

[8] S. Cerveny et al., J. Chem. Phys. 124, 194501 (2006).

[9] M. Y. Sun, S. Pejanovic, and J. Mijovic, Macromolecules 38, 
9854 (2005).

[10] N. Shinyashiki et al., J. Phys.: Condens. Matter 19, 205113 (2007).

[11] H. Vogel, Phys. Z. 22, 645 (1921); G. S. Fulcher, J. Am. Chem. Soc. 8, 339 (1925); Phys. Z. 8, 789 (1925); G. Tammann and W. Hesse, Z. Anorg. Allg. Chem. 156, 245 (1926).

[12] S. Cerveny, J. Colmenero, and A. Alegría, J. Non-Cryst. Solids 353, 4523 (2007).

[13] S. Cerveny, J. Colmenero, and A. Alegría, Macromolecules 38, 7056 (2005).

[14] S. Cerveny, J. Colmenero, and A. Alegría, Phys. Rev. Lett. 97, 189802 (2006).

[15] B. Bagchi, Chem. Rev. (Washington, D.C.) 105, 3197 (2005).

[16] A. Knocks and H. Weingartner, J. Phys. Chem. B 105, 3635 (2001).

[17] M. Tyagy and S. S. N. Murthy, Carbohydr. Res. 341, 650 (2006).

[18] S. Takashima, Biophys. J. 46, 29 (1984).

[19] N. Shinyashiki et al., J. Chem. Phys. 107, 6877 (1996).

[20] S. Havriliak and S. Negami, Polymer 8, 161 (1967).

[21] R. H. Cole and K. S. Cole, J. Chem. Phys. 10, 98 (1942).

[22] T. X. Xiang and B. D. Anderson, Pharm. Res. 22, 1205 (2005).

[23] V. Molinero, T. Cagin, and W. A. Goddard, J. Phys. Chem. A 108, 3699 (2004)
[24] C. J. Roberts and P. G. Debenedetti, J. Phys. Chem. B 103, 7308 (1999).

[25] S. Boresch, P. Hochtl, and O. Steinhauser, J. Phys. Chem. B 104, 8743 (2000)

[26] H. Jansson and J. Swenson, Eur. Phys. J. E 12, S51 (2003).

[27] P. Berntsen et al., Biophys. J. 89, 3120 (2005).

[28] C. Lorthioir, A. Alegria, and J. Colmenero, Phys. Rev. E 68, 031805 (2003).

[29] A. C. Genix, A. Arbe, F. Alvarez, J. Colmenero, L. Willner, and D. Richter, Phys. Rev. E 72, 031808 (2005).

[30] D. Cangialosi et al., J. Chem. Phys. 123, 144908 (2005).

[31] G. A. Schwartz, J. Colmenero, and A. Alegría, Macromolecules 40, 3246 (2007).

[32] H. Tanaka, J. Phys.: Condens. Matter 12, R207 (2000).

[33] F. Mizuno et al., J. Non-Cryst. Solids 352, 5147 (2006).

[34] C. A. Angell, J. Non-Cryst. Solids 131-133, 13 (1991).

[35] C. A. Angell, J. Phys. Chem. 97, 6339 (1993).

[36] P. W. Fenimore et al., Proc. Natl. Acad. Sci. U.S.A. 99, 16047 (2002).

[37] J. Swenson, H. Jansson, and R. Bergman, Phys. Rev. Lett. 96, 247802 (2006)

[38] T. E. Dirama, J. E. Curtis, G. A. Carri, and A. P. Sokolov, J. Chem. Phys. 124, 034901 (2006). 